\title{
A NEW BREAKTHROUGH IN LINEAR-SYSTEM THEORY: KHARITONOV'S RESULT.
}

\author{
P. P. Vaidyanathan. \\ Department of Electrical Engineering, California Institute of Technology \\ Pasadena, CA 91125
}

\begin{abstract}
Given a real coefficient polynomial $D(s)$, there exist several procedures for testing whether it is strictly Hurwitz (i.e., whether it has all its zeros in the open left-half plane). If the coefficients of $D(s)$ are uncertain and belong to a known interval, such testing becomes more complicated because there is an infinitely large family of polynomials to which $D(s)$ now belongs. It was shown by Kharitonov that in this case it is necessary and sufficient to test only four polynomials in order to know whether every polynomial in the family is strictly Hurwitz. An interpretation of this result in terms of reactance functions (i.e., LC impedances) was recently proposed. These results were also extended recently for the testing of positive real property of rational transfer functions with uncertain denominators. In this paper we review these results along with detailed proofs and discuss extensions to the discrete-time case.
\end{abstract}

\section{INTRODUCTION.}

Consider a continuous-time linear time invariant system characterized by a transfer function $H(s)=\mathcal{N}(s) / D(s)$ where $\mathcal{N}(s)$ and $D(s)$ are polynomials in $s$. We shall assume that there are no uncanceled common factors between $\mathcal{N}(s)$ and $D(s)$. The system $H(s)$ is said to be boundedinput bounded-output stable (or just BIBO stable $[1,2]$ ) if every bounded input $x(t)$ produces a bounded output $y(t)$. It is well-known that the system is BIBO stable if and only if all its poles (which are the zeros of $D(s)$ ) have strictly negative real parts. Now the polynomial $D(s)$ is said to be strictly Hurwitz (abbreviated SH) if all its zeros are in the open left half plane (abbreviated LHP), i.e., if each zero $s_{k}$ satisfies $\operatorname{Re}\left[s_{k}\right]<0$. Thus, the system $H(s)$ is BIBO stable if and only if $D(s)$ is SH. (In this paper, we shall imply BIBO stability even if we loosely use the word 'stable'.) In this paper we shall be concerned with tests for the SH property under uncertain conditions.

The polynomial $D(s)$ has the form

$$
D(s)=d_{0}+d_{1} s+\ldots+d_{N} s^{N}
$$

and we shall assume that the coefficients $d_{n}$ are real. With $d_{N} \neq 0$, the degree of $D(s)$ is equal to $N$. If all the coeff-

Work supported in parts by the National Science Foundation grants DCI 8552579 and MIP 8604456. cients $d_{n}$ are known, then we can use standard procedures to test whether $D(s)$ is $\mathrm{SH}$, one of these being the wellknown Routh-Hurwitz criterion $[2,3]$, reviewed in Sec. II. If, on the other hand, the coefficients are uncertain, then the problem is more involved. Suppose, for example, that each coefficient belongs to an interval

$$
d_{n, m} \leq d_{n} \leq d_{n, M}, \quad 0 \leq n \leq N,
$$

and that the bounds $d_{n, m}$ and $d_{n, M}$ are known. We now have a family of polynomials of the form (1), and the coefficients of each member of this family satisfy (2). Based only on the knowledge of the bounds $d_{n, m}$ and $d_{n, M}$ for $0 \leq n \leq N$, we would like to answer the following question: is every member of this family SH?

No doubt, the first thought that occurs to us is that we have to test an infinite number of polynomials in order to know the answer. Kharitonov showed [4] in 1979 that it is sufficient to test only four polynomials! These four polynomials, called the Kharitonov polynomials, will be defined in a later section when we are mathematically ready for it. Even though Kharitonov's original proof was somewhat incomprehensible to the electical engineer, a second proof based on electrical reactances was given in [5]. In Sec. II of this paper, we shall first review the well-known connection between reactances [3] and SH-test procedures [2] (such as the Routh-Hurwitz criterion). Once the reader is reminded of this connection, the construction of Kharitonov polynomials and the proof of Kharitonov's claim follow in a straightforward manner (Sec. III). In Sec. IV we also review a number of extentions of Kharitonov's result for the case of discrete-time systems [6,7], including a new result for this case. These will be explained in Sec. $V$ based on discrete-time reactances, so that they do not come as a complete surprise. However, as discussed in Sec. IV, there is no exact equivalent of Kharitonov's result for the discretetime case. Kharitonov-type results for the test of other properties such as positive realness of a rational transfer function with uncertain coefficients (which find application in adaptive filtering) are reviewed in Sec. VI.

Notice the implicit assumption that the variations of the coefficients are independent of each other so that each coefficient can take on any value in the interval regardless of the values taken by the other coefficients. The intervals 
(2) can be visualized by imagining a hyper rectangle in the $(N+1)$-dimensional space of coefficients. Points that lie on or inside this rectangle describe the family of polynomials characterized by (2). The term 'interval polynomials' is sometimes used to describe the family of polynomials described in this manner.

Notations. The frequency variables for continuous time and discrete time cases will be, respectively, $\Omega$ and $\omega$, so that the 'steady state' of a transfer function is obtained by setting $s=j \Omega$ (continuous time case) and $z=e^{j \omega}$ (discrete time case). A polynomial with real coefficients is said to be a real polynomial. A real rational function is a ratio of two real polynomials. The degree of a rational function is equal to the highest power of $s$ (or $z^{-1}$ in the discrete case) appearing in the expression $H(s)$ (or $H(z)$ ) after canceling off any common factors.

\section{ROUTH-HURWITZ TESTS, REACTANCES, AND CONTINUED-FRACTION EXPANSIONS}

In this section, we assume that $d_{n}$ are known (i.e., no uncertainity), and review certain well-known procedures for testing the SH property of $D(s)$. The most obvious procedure wculd of course be to find all the $N$ zeros of $D(s)$ and test the real parts. This, however, is unnecessary work, and can sometimes lead to incorrect conclusions due to numerical errors.

2.1. Continued-fraction expansion and the SH-test.

A more elegant and efficient procedure is the RouthHurwitz test, described in many electrical engineering texts $[2,3]$. In this procedure, a table (called Routh's table) is formulated, and primarily by inspection of signs of certain entries in the table, it is possible to tell whether $D(s)$ is SH os not. We shall now describe this test using the language of reactances and continued fraction expansions.

Any polynomial $D(s)$ of the form (1) can be written in the form

$$
D(s)=P\left(s^{2}\right)+s Q\left(s^{2}\right)
$$

simply by collecting the terms with even powers of $s$ and the terms with odd powers of $s$ into two different groups. For example, if

$$
D(s)=2 s^{4}+s^{3}+8 s^{2}+2 s+6
$$

then

$$
P\left(s^{2}\right)=2 s^{4}+8 s^{2}+6, \quad Q\left(s^{2}\right)=s^{2}+2 .
$$

The coefficients of $P(s)$ and $Q(s)$ are given by

$$
p_{n}=d_{2 n}, \quad q_{n}=d_{2 n+1} .
$$

Suppose we now form a function

$$
F(s)=\frac{P\left(s^{2}\right)}{s Q\left(s^{2}\right)}
$$

and then perform a continued-fraction expansion (abbreviated CFE) $[3,8]$. $\ddagger$ By inspection of the coefficients of the CFE it is possible to tell whether $D(s)$ is $\mathrm{SH}$ or not. The first step in obtaining the CFE is to write it as

$$
F(s)=\frac{2 s^{4}+8 s^{2}+6}{s^{3}+2 s}=2 s+\frac{4 s^{2}+6}{s^{3}+2 s}
$$

i.e., simply divide the highest power in the numerator by the highest power in the denominator to obtain $2 s$. Then write $F(s)=2 s+F_{1}(s)$. Since $F_{1}(s)$ has numerator degree less than denominator degree, we can repeat the operation on $1 / F_{1}(s)$ :

$$
\frac{1}{F_{1}}(s)=\frac{1}{4} s+F_{2}(s)
$$

Continuing in this manner we obtain the CFE

$$
F(s)=\frac{2 s^{4}+8 s^{2}+6}{s^{3}+2 s}=2 s+\frac{1}{\frac{1}{4} s+\frac{1}{8 s+\frac{1}{\frac{1}{12} s}}}
$$

More generally if $D(s)$ is as in (1), then the CFE looks like

$$
F(s)=\frac{P\left(s^{2}\right)}{s Q\left(s^{2}\right)}=L_{1} s+\frac{1}{C_{2} s+\frac{1}{L_{3} s+\frac{1}{C_{4} s+\frac{1}{L_{5} s+\ldots}}}}
$$

If the degree of $s Q\left(s^{2}\right)$ exceeds that of $P\left(s^{2}\right)$ then the first coefficient $L_{1}$ turns out to be zero, in which case we have to re-define $F(s)$ to be $s Q\left(s^{2}\right) / P\left(s^{2}\right)$ before performing the CFE. The quantities $L_{1}, C_{2}, \ldots$ etc. in the CFE of $F(s)$ are called the coefficients of the CFE. There are $N$ such coeffcients if $D(s)$ has degree $N$. It is well-known $[3,8]$ that $D(s)$ is SH if and only if these $N$ CFE coefficients are strictly positive.

One can attach a physical meaning for the CFE coefficients by considering the electrical network (LC network) of Fig. 1. If the inductors and capacitors in this network are assigned values equal to the CFE coefficients of $F(s)$, then the input impedance of the network is precisely equal to $F(s)$. We know that the network is passive (in fact lossless) if and only if all the $\mathrm{L}, \mathrm{C}$ elements are positive. The input impedance of an LC network with positive element-values is called a reactance.

We can summarize these ideas as follows:

Lemma 2.1. Given the polynomial $D(s)$, form the func-

$¥$ if the degree of $P\left(s^{2}\right)$ is less than that of $s Q\left(s^{2}\right)$, then we define $F(s)=s Q\left(s^{2}\right) / P\left(s^{2}\right)$. 
tion $F(s)$ defined to be $P\left(s^{2}\right) / s Q\left(s^{2}\right)$ or $s Q\left(s^{2}\right) / P\left(s^{2}\right)$ as described earlier. Then the following are true:

1. $D(s)$ is $\mathrm{SH}$ if and only if $F(s)$ is a reactance of degree $N$.

2. The function $F(s)$, in turn, is a reactance of degree $N$ if and only if all the $N$ CFE coefficients are strictly positive.

3. The function $F(s)$ is a reactance of degree $N$ if and only if it is the input impedance of a network of the form in Fig. 1 with all the $N \mathrm{~L}, \mathrm{C}$ elements strictly positive.

In practice, the above CFE is performed in a more efficient way, by means of a neat tabulation which reduces the amount of writing and paper-work required to do the CFE, and also leads to simple computer programs. This tabulation is the Routh array [2]. For the above example, this array is shown in Fig. 2. The first and the second rows represent the coefficients of $P(s)$ and $Q(s)$ respectively. After this the elements of the successive rows represent the numerators of $F_{1}(s), F_{2}(s)$ and so on. These coefficients can be generated by a simple cross-multiplication operation, demonstrated, for example, in [2, page 324]. Once the table is formed in this way, the first column bears all the information about the CFE. More specifically, if we take the ratio between successive entries of the first column, we obtain the CFE coefficients. In our example these ratios are $2 / 1,1 / 4,8$, and $1 / 12$ which are precisely the CFE coefficients in (10).

\subsection{Review of properties of reactances.}

The proof of the above connection between CFEs, reactances, and SH-tests is based on a number of crucial properties of a reactance. Even though we shall skip this proof (see $[3,8])$, we would like to review these properties here.

1. The poles and zeros of a reactance are all single (i.e., multiplicity one) and are located on the $j \Omega$ axis (i.e., imaginary axis) of the $s$ plane. Moreover the poles and zeros are interlaced (Fig. 3).

2. Consequently a reactance is always of the form $F(s)=$ $P\left(s^{2}\right) / s Q\left(s^{2}\right)$ or $s Q\left(s^{2}\right) / P\left(s^{2}\right)$ where $P(s)$ and $Q(s)$ are real-coefficient polynomials. Another consequence is that there is a pole or zero at $s=0$ and also at $s=\infty$.

3. As a result of Property $2, F(j \Omega)$ is purely imaginary, i.e., $F(j \Omega)=j V(\Omega)$ where $V(\Omega)$ is a real-valued function. Since the coefficients of $F(s)$ happen to be real, $V(\Omega)$ is an odd function, i.e., $V(\Omega)=-V(-\Omega)$.

4. A plot of $V(\Omega)$ has discontinuities at the poles, and is monotone increasing elsewhere as shown in Fig. 4.

Conversely [3], given a function $F(s)$, we can test whether it is a reactance by studying Property 1 . More specifically, we have:

Lemma 2.2. Suppose $F(s)$ is a real rational function such that a) all poles and zeros are single, b) they are constrained to lie on the imaginary axis, and c)poles and zeros are interlaced. Then either $F(s)$ or $-F(s)$ is a reactance.
We restate this lemma in terms of plots of the type in Fig. 4, for better visualization.

Lemma 2.3 Let $F(s)$ be a real rational function of degree $N$ of the form $P\left(s^{2}\right) / s Q\left(s^{2}\right)$ where $P(s)$ and $Q(s)$ are polynomials in $s$ (and with no common factors) so that $F(j \Omega)=j V(\Omega)$ with $V(\Omega)$ real. If the plot of $V(\Omega)$ has $N$ distinct singularities, and if it is monotone increasing between singularities (with a zero interlaced between adjacent singularities) then $F(s)$ is a reactance.

If $F(s)$ satisfies the conditions of the Lemma, then $D(s)$ defined as in (3) is SH. This Lemma is unbeleivably powerful as we shall see.

\section{STATEMENT AND PROOF OF KHARITONOV'S THEOREM}

Now consider the family of polynomials $D(s)$ with the coefficients $d_{n}$ belonging to the independent intervals as in (2). Define $P\left(s^{2}\right)$ and $Q\left(s^{2}\right)$ as usual, as in (3). Note that $P\left(s^{2}\right)$ has even-numbered coefficients $d_{2 n}$ and $Q\left(s^{2}\right)$ has odd numbered coefficients $d_{2 n+1}$. Assuming that the coefficients of $D(s)$ can have any values in the intervals defined by (2), we have a family of polynomials with infinite number of members. Those polynornials in this family for which each of the coefficients $d_{n}$ takes on an extreme value (i.e., $d_{n, m}$ or $\left.d_{n, M}\right)$ are called vertex polynomials. In general we have a total of $2^{N+1}$ vertex polynomials. For example if $N=2$, there are three coefficients, and we have $2^{3}=8$ ver-

tex polynomials as demonstrated in Fig. 5. In Kharitonov's theorem, four of these $2^{N+1}$ vertex polynomials play a crucial role. Our first aim here is therefore to define these four polynomials.

Suppose we define a polynomial $P_{1}\left(s^{2}\right)$ by choosing the successive coefficients of $P\left(s^{2}\right)$ to be: $\min , \max , \min , \max$, ... and so on. In other words let

$$
P_{1}\left(s^{2}\right)=p_{0, m}+p_{1, M} s^{2}+p_{2, m} s^{4}+p_{3, M} s^{6}+\ldots
$$

Similarly define $P_{2}\left(s^{2}\right)$ in exactly the opposite way (i.e., $\max , \min , \max , \min , \ldots$ etc.):

$$
P_{2}\left(s^{2}\right)=p_{0, M}+p_{1, m} s^{2}+p_{2, M} s^{4}+p_{3, m} s^{6}+\ldots
$$

In the same way let us define $Q_{1}\left(s^{2}\right)$ and $Q_{2}\left(s^{2}\right)$ :

$$
\begin{aligned}
& Q_{1}\left(s^{2}\right)=q_{0, m}+q_{1, M} s^{2}+q_{2, m} s^{4}+q_{3, M} s^{6}+\ldots \\
& Q_{2}\left(s^{2}\right)=q_{0, M}+q_{1, m} s^{2}+q_{2, M} s^{4}+q_{3, m} s^{6}+\ldots
\end{aligned}
$$

We can now define four specific choices of the polynomial $D(s)$ by combining the two specific choices for $P\left(s^{2}\right)$ and the two for $Q\left(s^{2}\right)$. These are given by

$$
D_{k n}(s)=P_{k}\left(s^{2}\right)+s Q_{n}\left(s^{2}\right), \quad 1 \leq k, n \leq 2 .
$$

These four polynomials $D_{11}(s), D_{12}(s), D_{21}(s), D_{22}(s)$ are called the Kharitonov polynomials. We are now ready to state the main result: 
Theorem 3.1. (Kharitonov's theorem.) The real coefficient polynomial $D(s)$ is strictly Hurwitz for every possible set of values of coefficients belonging to the interval (2) if and only if the four vertex polynomials $D_{k n}, 1 \leq k, n \leq 2$ are strictly Hurwitz.

In order to prove this theorem, we shall first assume that the coefficients of $Q(s)$ (i.e., $d_{2 n+1}$ ) are fixed so that only the coefficients $p_{n}$ of $\left.P(s)\right)$ are uncertain, belonging to the range

$$
p_{n, m} \leq p_{n} \leq p_{n, M} .
$$

The family of polynomials, of course, still has an infinite number of members. The four polynomials in (16) now degenerate into only two polynomials,

$$
\begin{aligned}
& D_{1}(s)=P_{1}\left(s^{2}\right)+s Q\left(s^{2}\right) \\
& D_{2}(s)=P_{2}\left(s^{2}\right)+s Q\left(s^{2}\right)
\end{aligned}
$$

and an arbitrary member of the infinite family has the form

$$
D(s)=P\left(s^{2}\right)+s Q\left(s^{2}\right) .
$$

Our aim now is to prove that if $D_{1}(s)$ and $D_{2}(s)$ are SH then $D(s)$ is SH for every $p_{n}$ satisfying (17). This is equivalent to proving that $P\left(s^{2}\right) / s Q\left(s^{2}\right)$ is a reactance of degree $N$ if $P_{1}\left(s^{2}\right) / s Q\left(s^{2}\right)$ and $P_{2}\left(s^{2}\right) / s Q\left(s^{2}\right)$ are reactances of degree $N$.

For this, note that if we evaluate the polynomials $P_{1}\left(s^{2}\right), P_{2}\left(s^{2}\right)$ and $P\left(s^{2}\right)$ for $s=j \Omega$ we get

$$
\begin{gathered}
P_{1}\left(-\Omega^{2}\right)=p_{0, m}-p_{1, M} \Omega^{2}+p_{2, m} \Omega^{4}-p_{3, M} \Omega^{6}+\ldots \\
P\left(-\Omega^{2}\right)=p_{0}-p_{1} \Omega^{2}+p_{2} \Omega^{4}-p_{3} \Omega^{6}+\ldots \\
P_{2}\left(-\Omega^{2}\right)=p_{0, M}-p_{1, m} \Omega^{2}+p_{2, M} \Omega^{4}-p_{3, m} \Omega^{6}+\ldots
\end{gathered}
$$

From (17) we know that $p_{n}$ is sandwiched between $p_{n, m}$ añd $p_{n, M}$. And since $\Omega^{2} \geq 0$, it is immediately clear from (21-23) that $P\left(-\Omega^{2}\right)$ is sandwiched between $P_{1}\left(-\Omega^{2}\right)$ and $P_{2}\left(-\Omega^{2}\right)$, i.e.,

$$
P_{1}\left(-\Omega^{2}\right) \leq P\left(-\Omega^{2}\right) \leq P_{2}\left(-\Omega^{2}\right), \quad \text { for all } \Omega .
$$

This property is the key to the secret of Kharitonov's result. Everything else follows from this, as we shall see next.

Let us denote

$$
F(s)=\frac{P\left(s^{2}\right)}{s Q\left(s^{2}\right)}, F_{1}(s)=\frac{P_{1}\left(s^{2}\right)}{s Q\left(s^{2}\right)}, F_{2}(s)=\frac{P_{2}\left(s^{2}\right)}{s Q\left(s^{2}\right)} .
$$

For $s=j \Omega$, the quantities $F(s), F_{1}(s)$ and $F_{2}(s)$ are purely imaginary so that we can write

$$
F(j \Omega)=j V(\Omega), \quad F_{1}(j \Omega)=j V_{1}(\Omega), \quad F_{2}(j \Omega)=j V_{2}(\Omega) .
$$

Assuming $D_{1}(s)$ and $D_{2}(s)$ to be SH of degrees $N, F_{1}(s)$ and $F_{2}(s)$ are reactances of degree $N$. Moreover these reactances have poles at $s=0$. It is also obvious that $F(s), F_{1}(s)$ and $F_{2}(s)$ have the same set of poles so that $V(\Omega), V_{1}(\Omega)$ and $V_{2}(\Omega)$ 'blow up' for the same set of values of $\Omega$. Summarizing we have the following situation.

1. $F_{1}(s)$ and $F_{2}(s)$ are reactances with the same set of poles. Moreover $F(s)$ has this same set of poles so that $V_{1}(\Omega), V_{2}(\Omega)$ and $V(\Omega)$ 'blow up' at the same set of frequencies.

2. The numerator of $V(\Omega)$ is sandwiched between those of $V_{1}(\Omega)$ and $V_{2}(\Omega)$ because of (24). The deominators of $V(\Omega), V_{1}(\Omega)$ and $V_{2}(\Omega)$ are the same, viz., $\Omega Q\left(-\Omega^{2}\right)$.

3. In addition to this, $F_{1}(s)$ and $F_{2}(s)$ are reactances with a pole at $s=0$.

4. As a consequence of these the plots of $V_{1}(\Omega)$ and $V_{2}(\Omega)$ are as shown in Fig. 6.

5. In view of these, the only possibile behavior of $V(\Omega)$ is as indicated in Fig. 6, i.e., it has $N$ singularities in the range $-\infty \leq \Omega \leq \infty$, is monotone increasing between singularities and has a zero in between each pair of singularities. By using Lemma 2.3 we can therefore conclude that $F(s)$ is a reactance so that $D(s)$ is SH!

We have therfore proved Kharitonov's theorem assuming that only the even-numbered coefficients $d_{2 n}$ are uncertain. We shall state this as a Lemma for ease of reference

Lemma 3.1. Let $D(s)$ be a real-coefficient polynomial of degree $N$ whose even-numbered coefficients $d_{2 n}$ (equal to $p_{n}$ ) are uncertain, belonging to the interval (17). Define the two vertex polynomials $D_{1}(s)$ and $D_{2}(s)$ as in $(18,19)$. Then $D(s)$ is SH for all $p_{n}$ satisfying (17) if and only if $D_{1}(s)$ and $D_{2}(s)$ are $\mathrm{SH}$.

In our above discussions, we have proved the 'if' part of the Lemma. The 'only if' part follows trivially because $D_{1}(s)$ and $D_{2}(s)$ are themselves members of the family of polynomials under discussion.

The next logical step now is to assume that the coefficients of $P\left(s^{2}\right)$ are fixed and those of $Q\left(s^{2}\right)$ are uncertain. In other words, only the odd numbered coefficients $d_{2 n+1}$ of $D(s)$ are uncertain. By following an argument very similar to the above, we can arrive at the following Lemma. Details are omitted to avoid repetitions.

Lemma 3.2. Let $D(s)$ be a real-coefficient polynomial of degree $N$ whose odd-numbered coefficients $d_{2 n+1}$ (equal to $\left.q_{n}\right)$ are uncertain, belonging to the interval

$$
q_{n, m} \leq q_{n} \leq q_{n, M}
$$

Define the two vertex polynomials $D_{3}(s)$ and $D_{4}(s)$ as

$$
\begin{gathered}
D_{3}(s)=P\left(s^{2}\right)+s Q_{1}\left(s^{2}\right) \\
D_{4}(s)=P\left(s^{2}\right)+s Q_{2}\left(s^{2}\right) .
\end{gathered}
$$


Then $D(s)$ is SH for every $q_{\mathrm{n}}$ belonging to (27) if and only if $D_{3}(s)$ and $D_{4}(s)$ are $\mathrm{SH}$.

It is a simple matter to combine Lemmas 3.1 and 3.2 to obtain a proof of Kharitonov's theorem. For this assume that all the coefficients $d_{n}$ are uncertain, and assume that the four polynomials in (16) are SH. Since $D_{11}(s)$ and $D_{21}(s)$ are $\mathrm{SH}$, then by Lemma 3.1 we see that

$$
D_{a}(s)=P\left(s^{2}\right)+s Q_{1}\left(s^{2}\right)
$$

is $\mathrm{SH}$ for all $p_{n}$ in (17). Since $D_{12}(s)$ and $D_{22}(s)$ are $\mathrm{SH}^{-}$ the polynomial

$$
D_{b}(s)=P\left(s^{2}\right)+s Q_{2}\left(s^{2}\right)
$$

is $\mathrm{SH}$ for all $p_{n}$ in (17). Now by using Lemma 3.2 on the pair $D_{a}(s)$ and $D_{b}(s)$ we conclude that the polynomial $D(s)$ in (20) is SH for all $q_{n}$ in the range (27) for any $p_{n}$ in the range (17). In other words, $D(s)$ is SH for all $d_{n}$ in the range (2), which completes the proof of Kharitonov's theorem.

\section{EXTENSIONS TO THE DISCRETE-TIME CASE.}

As soon as Kharitonov's results were announced, it aroused great interest in the Engineering community. The natural question that came up was: can we get a similar result for discrete-time systems? People sat down and tried to prove a similar result, but it was soon found out that a straight-forward extension does not exist, as proved by certain counter examples $[6]$. However, some modified and partial results have been proved to be true, and in this section we shall elaborate on some of these.

\subsection{Review of basics.}

Consider a discrete-time linear time invariant (LTI) system characterized by a rational transfer function $H(z)=$ $\mathcal{N}(z) / D(z)$. We shall assume that this is a causal system. Here $\mathcal{N}(z)$ and $D(z)$ are polynomials in $z^{-1}$ with no common factors. Since $\mathcal{N}(z)$ does not affect stability properties, we can ignore its presence from now on. Let

$$
D(z)=d_{0}+d_{1} z^{-1}+\ldots+d_{N} z^{-N}
$$

with $d_{N} \neq 0$ so that the degree of $D(z)$ is equal to $N$. We say that $D(z)$ is strictly Hurw.tz (SH) if all its zeros are strictly inside the unit circle ot the $z$-plane. In other words, if $z_{k}$ is $z$ zero then $\left|z_{k}\right|<1$. It is well-known [9] that $H(z)$ is BIBO stable if and oxly if $D(z)$ is SH. The most commonly used procedure to test whether $D(z)$ is SH is the Jury-Marden test [10],\{11]. A detailed review of this and other tests along with structural interpretations can be found in [12]. These tests are applicable if all the coefficients $d_{n}$ are known and fixed

We shall restrict ourselves to the case where $d_{n}$ are real. The complex case can be handled by incorporating more complicated notations. Now assure that the coefficients belong to an interval as in (2). This means that we have a family of an infinite number of poly zomials. Once again we can define the $2^{N+1}$ vertex polynomials as we did in Sec. III. Is it possible to find a subset of these polynomials such that if each member in this subset is SH then every $D(z)$ belonging to the family is SH? The answer is, in general, no! In other words, there does not exist such a subset of vertex polynomials.

This is easily proved with the help of a counter example [6]: let $D(z)=1+d_{1} z^{-1}+1.35 z^{-2}+0.243 z^{-3}-0.2916 z^{-4}$, with

$$
-2.3 \leq d_{1} \leq 1.7 \text {. }
$$

In other words, all the coefficients are fixed except $d_{1}$ which is uncertain. There are only two vertex polynomials in this case, one of them corresponding to $d_{1}=-2.3$ and the other to $d_{1}=1.7$. It can be verified by use of standard tests [12] that these two vertex polynomials are $\mathrm{SH}$. However, the polynomial with $d_{1}=-1.3$ which belongs to (32), is not SH.

4.2. Extensions when only some coefficients are uncertain.

The good news now is that there are some special situations which permit an extension. For example, suppose all the coefficients $d_{n}$ are fixed, except only one coefficient $d_{K}$, where $K \geq N / 2$. Assume that $d_{K}$ belongs to a real interval so that

$$
d_{K, m} \leq d_{K} \leq d_{K, M}
$$

where the bounds $d_{K_{1} m}$ and $d_{K, M}$ are known. If we define $D_{1}(z)$ and $D_{2}(z)$ to be the vertex polynomials corresponding to $d_{K}=d_{K, m}$ and $d_{K}=d_{K, M}$ respectively, then we have the following result $[6]$.

Lemma 4.1. Let $D(z)$ be as in (31) with the $K$ th coefficient $d_{K}$ uncertain, with $K \geq N / 2$. Then $D(z)$ is SH for every possible value of $d_{K}$ in the interval (33) if and only if $D_{1}(z)$ and $D_{2}(z)$ are SH.

In fact, more is true. Suppose that the coefficients $d_{n}$ are fixed for $0 \leq n<N / 2$ and uncertain for $N / 2 \leq n \leq N$. In other words, let

$$
d_{n, m} \leq d_{n} \leq d_{n, M}, \quad N / 2 \leq n \leq N
$$

The number of uncertain coefficients depends on whether $N$ is even or odd. Clearly, this number is

$$
L= \begin{cases}\frac{N}{2}+1 & N \text { even } \\ \frac{N+1}{2} & N \text { odd }\end{cases}
$$

We can then define $2^{L}$ vertex polynomials by assigning one of the two extreme values to each of the uncertain coefficients. In this case we have the following result:

Lemma 4.2. The polynomial $D(z)$ is $\mathrm{SH}$ for all values of the $L$ uncertain coefficients if and only if the $2^{L}$ vertex polynomials are $\mathrm{SH}$.

So the generalization to the discrete case is different from the continuous-time case in two ways: first, only the last $L$ out of the $N+1$ coefficients are allowed to be uncertain. Second, the number of vertex polynomials to be 
tested is $2^{L}$ which grows exponentially with $N$. This is unlike in the continuous-time case where we had to test only four polynomials regardless of how large $N$ was.

\subsection{A closer discrete-time counterpart.}

Instead of working directly with the $N+1$ coefficients of $D(z)$ if we work with a modified set of $N+1$ coefficients then we have much better luck. In fact we can now get a very close analog of the continuous-time theorem in the sense that we have to perform only four tests in order to know whether the complete family of polynomials is SH. In this subsection we shall state this result.

The basic ingredient of the result to be stated holds whether $N$ is even or odd but the notational details are slightly different. We shall therefore assume that $N$ is even just as a matter of convenience and define the integer $M=$ $N / 2$. We shall define the mirror image of $D(z)$ to be the polynomial

$$
\hat{D}(z)=z^{-N} D\left(z^{-1}\right)=d_{N}+d_{N-1} z^{-1}+\ldots+d_{0} z^{-N} .
$$

Now define the two new polynomials

$$
S(z)=z^{M}[D(z)+\hat{D}(z)], \quad A(z)=z^{M}[D(z)-\hat{D}(z)]
$$

so that $S(z)$ is a symmetric polynomial and $A(z)$ is an antisymmetric polynomial. In other words, with

$$
S(z)=\sum_{n=-M}^{M} s_{n} z^{-n}, \quad A(z)=\sum_{n=-M}^{M} a_{n} z^{-n}
$$

the sequences $s_{n}$ and $a_{n}$ satisfy

$$
s_{n}=s_{-n}, \quad a_{n}=-a_{-n} .
$$

It is clear that we can express $D(z)$ as

$$
D(z)=z^{-M} \frac{S(z)+A(z)}{2}
$$

so that the sequences $\left\{s_{n} / 2\right\}$ and $\left\{a_{n} / 2\right\}$ are merely the even and odd parts $[9]$ of the sequence $\left\{d_{n}\right\}$. Because of the property (39), the Fourier transforms $S\left(e^{j \omega}\right)$ and $A\left(e^{j \omega}\right)$ are purely real and purely imaginary respectively (remembering that $d_{n}$ are real). To be more specific, we can write $[13],[14$, Sec. IV.A $]$

$$
\begin{gathered}
S\left(e^{j \omega}\right)=\sum_{n=0}^{M} \hat{s}_{n} \cos (n \omega), \\
A\left(e^{j \omega}\right)=j \sin (\omega) \sum_{n=0}^{M-1} \hat{a}_{n} \cos (n \omega) .
\end{gathered}
$$

Now it is well-known that $\cos (n \omega)$ can be expressed as a polynomial in $\cos (\omega)$. In fact, we have [14, page 101]

$$
\cos (n \omega)=\sum_{k=0}^{n} c_{k, n} \cos ^{k}(\omega)
$$

where $c_{k, n}$ are the coefficients of the $n$th degree Chebyshev polynomial [15]. The quantity $\cos \omega \operatorname{can}$ in turn be expressed as $\cos \omega=2 \cos ^{2}(\omega / 2)-1$ so that we obtain the following representation for $D\left(e^{j \omega}\right)$.

$$
D\left(e^{j \omega}\right)=e^{-j \omega M}[P(\omega)+j \sin (\omega) Q(\omega)]
$$

where

$$
P(\omega)=\sum_{n=0}^{M} p_{n} \cos ^{2 n}(\omega / 2), \quad Q(\omega)=\sum_{n=0}^{M-1} q_{n} \cos ^{2 n}(\omega / 2)
$$

where $p_{n}$ and $q_{n}$ are real-valued coefficients. Summarizing, we have converted the set of $N+1$ coefficients $d_{n}$ into the set of coefficients $p_{n}\left(M+1\right.$ of them) and $q_{n}$ ( $M$ of them).

The uncertainty of the coefficients $d_{n}$ reflects as uncertainty of the coefficients $p_{n}$ and $g_{n}$. Suppose we assume that $p_{n}$ and $q_{n}$ belong to the intervals,

$$
p_{n, m} \leq p_{n} \leq p_{n, M}, \quad 0 \leq n \leq M
$$

and

$$
q_{n, m} \leq q_{n} \leq q_{n, M}, \quad 0 \leq n \leq M-1 .
$$

This does not, however mean that the coefficients $d_{n}$ belong to intervals like (2) because the bounds on various $d_{n}$ 's are not independent anymore. In this sense, we are not dealing with an interval polynomial any more (see Sec. I). In other words, the region from which the coefficients $d_{n}$ are drawn is not a hyper-rectangle (unlike in Sec. I), so that we are dealing with a totally different type of uncertainty.

In order to state the extended version of Kharitonov's theorem we now define the vertex polynomials

$$
\begin{aligned}
& P_{1}(\omega)=\sum_{n=0}^{M} p_{n, m} \cos ^{2 n}(\omega / 2), \\
& P_{2}(\omega)=\sum_{n=0}^{M} p_{n, M} \cos ^{2 n}(\omega / 2), \\
& Q_{1}(\omega)=\sum_{n=0}^{M-1} q_{n, m} \cos ^{2 n}(\omega / 2), \\
& Q_{2}(\omega)=\sum_{n=0}^{M-1} q_{n, M} \cos ^{2 n}(\omega / 2)
\end{aligned}
$$

and define the four $D(z)$ polynomials according to

$$
D_{k n}\left(e^{j \omega}\right)=e^{-j \omega M}\left[P_{k}(\omega)+j \sin (\omega) Q_{n}(\omega)\right],
$$

with $1 \leq k, n \leq 2$. We then have the following result, to be proved in Sec. $V$.

Theorem 4.1 Every $D(z)$ with coefficients of $p_{n}$ and $q_{n}$ constrained by (46),(47) is SH if and only if the four polynomials $D_{11}(z), D_{21}(z), D_{12}(z)$ and $D_{22}(z)$ are SH.

\section{PROOFS OF THE DISCRETE-TIME VERSIONS USING DISCRETE-TIME REACTANCES}


At first sight, the concept of a reactance does not seem to make sense in the discrete-time case because we do not have electrical LC networks in the discrete world. However, we can still mathematically define a reactance in this case, by use of the bilinear transformation. Given a transfer function $F(s)$, the bilinear transformation replaces $s$ with $\left(1-z^{-1}\right) /\left(1+z^{-1}\right)$ to obtain a new function $G(z)$. Clearly $G(z)$ is real rational if $F(s)$ is. The bilinear transformation maps the $s$-plane into the $z$-plane such that [9]

$$
|z| \begin{cases}=1 & \text { if } \operatorname{Re}[s]=0 \\ <1 & \text { if } \operatorname{Re}[s]<0 \\ >1 & \text { if } \operatorname{Re}[s]>0\end{cases}
$$

In particular therefore, the imaginary axis is mapped onto the unit circle. If, for example, $F(s)$ is a reactance, then the behaviour of $G\left(e^{j \omega}\right)$ in the region $-\pi \leq \omega \leq \pi$ is analogous to the behavior of $F(j \Omega)$ in the region $-\infty \leq \Omega \leq \infty$.

\subsection{Discrete-time reactances.}

A discrete-time reactance $G(z)$ is merely a bilinearly transformed version of a continuous-time reactance $F(s)$. Its importance in connection with the $\mathrm{SH}$ property has been recognized earlier [16]. All the $s$-domain properties of reactances mentioned in Sec. II get transformed into the $z$-domain in an obvious manner. For example all the poles and zeros are single, restricted to the unit circle, and are interlaced with each other. Also, $G\left(e^{j \omega}\right)$ is purely imaginary i.e., $G\left(e^{j \omega}\right)=j V(\omega)$ where $V(\omega)$ is real-valued for all $\omega$. The function $V(\omega)$ becomes infinite exactly $N$ times in the range $0 \leq \omega<2 \pi$ where $N$ is the degree of the reactance. Thus if we plot $V(\omega)$ for $0 \leq \omega<2 \pi$, then the plot 'blows up' exactly $N$ times, is monotone increasing between these singularites, and is equal to zero once between each pair of singularities. All these properties are summarized in the examples shown in Fig. 7. Note that a reactance has to have a pole or zero at $z=1$. Same is true at $z=-1$. The following theorem is the discrete-time counterpart of some of the-results mentioned in Sec. II.

Theorem 5.1 A real rational $G(z)$ of degree $N$ is a reactance if and only if $G\left(e^{j \omega}\right)=j V(\omega)$ where $V(\omega)$ is a real valued function with the following features: a) it has $N$ singularities in $0 \leq \omega<2 \pi, b)$ it is monotone increasing between singularities so that it has a zero between every pair of singularities.

Notice that if $G(z)$ is a reactance then so is $\alpha G(z)$ for any $\alpha>0$. Moreover the sum of reactances is a reactance. Both of these can be verified by use of the above theorem. As a consequence, we have the following result:

Corollary. Let $G_{1}(z)$ and $G_{2}(z)$ be reactances. Then $G(z) \triangleq \lambda G_{1}(z)+(1-\lambda) G_{2}(z)$ is a reactance for $0 \leq \lambda \leq 1$.

5.2. Relation between discrete-time reactances and the $S H$ property.

Not surprisingly, the SH property of $D(z)$ is equivalent to the reactance property of a closely related rational function. This connection is given by the following lemma. Lernma 5.1 Let $D(z)$ be a real-coefficient polynomial of the form (31) with $d_{N} \neq 0$. Define the real rational function

$$
G(z)=\frac{D(z)+z^{-L} \widehat{D}(z)}{D(z)-z^{-L} \widehat{D}(z)}
$$

where $L$ is an arbitrary non-negative integer, and $\widehat{D}(z)$ is the mirror image of $D(z)$ defined in (36). Then $D(z)$ is $\mathrm{SH}$ if and only if $G(z)$ is a reactance of degree $N+L$.

To prove the 'only if' part, assume that $D(z)$ is SH so that all its zeros are strictly inside the unit circle. Consider the ratio

$$
H(z) \triangleq \frac{\widehat{D}(z)}{D(z)}
$$

which is BIBO stable because $D(z)$ is $\mathrm{SH}$. This is a rational function of degree $N$ because there can be no common zeros between $D(z)$ and $\widehat{D}(z) .{ }^{\ddagger}$ Moreover it is an allpass function because

$$
\left|\widehat{D}\left(e^{j \omega}\right)\right|=\left|e^{-j \omega N} D\left(e^{-j \omega}\right)\right|=\left|D\left(e^{j \omega}\right)\right|
$$

so that $\left|H\left(e^{j \omega}\right)\right|=1$. In other words $H(z)$ is a real rational stable allpass function of degree $N$ so that $z^{-L} H(z)$ is a stable allpass function of degree $L+N$.

Now it is well-known [17] that the phase response of a $K$ th degree stable allpass function is monotone decreasing, and spans a range of $2 \pi K$ as $\omega$ changes from 0 to $2 \pi$. From (54) we obtain

$$
G\left(e^{j \omega}\right)=\frac{1+e^{j \phi(\omega)}}{1-e^{j \phi(\omega)}}=-j \cot \left[\frac{\phi(\omega)}{2}\right]
$$

where $\phi(\omega)$ is monotone decreasing, spanning a range of $2 \pi(L+N)$. We therefore see that $G\left(e^{j \omega}\right)$ can be written as $j V(\omega)$ where $V(\omega)$ is a real function which has $N+L$ singularities in the range $0 \leq \omega<2 \pi$, and in between adjacent singularities there is precisely one zero. Because of the nature of the cotangent function, $V(\omega)$ is monotone between singularities.

Summarizing, we see that $G(z)$ is a reactance function of degree $N+L$, proving the 'only if' part of the Lemma. The 'if' part can be proved essentially by carrying out the argument backwards.

\subsection{Proofs of Lemmas 4.1 and 4.2}

We shall first prove Lemma 4.1 using Lemma 5.1. Assume, accordingly, that $D_{1}(z)$ and $D_{2}(z)$ defined in Lemma 5.1 are $\mathrm{SH}$. The aim is to prove that $D(z)$ is $\mathrm{SH}$ for all $d_{K}$ in the interval (33). Since $D_{1}(z)$ and $D_{2}(z)$ are SH, the quantities

$$
G_{1}(z)=\frac{D_{1}(z)+z^{-L} \widehat{D}_{1}(z)}{D_{1}(z)-z^{-L} \widehat{D}_{1}(z)}
$$

$\ddagger$ This is because the zeros of $\widehat{D}(z)$, which are reciprocals of those of $D(z)$ are located strictly outside the unit circle. 


$$
G_{2}(z)=\frac{D_{2}(z)+z^{-L} \widehat{D}_{2}(z)}{D_{2}(z)-z^{-L} \widehat{D}_{2}(z)}
$$

are reactances of degree $N$ for any non-negative integer $L$. Notice that the only difference between $D_{1}(z)$ and $D_{2}(z)$ is in the $K$ th coefficient. If we choose $L=2 K-N$ then the denominators of (58) and (59) are identical because the $K$ th coefficient $d_{K}$ cancels off. Only the numerators of (58) a ad (59) are now different. This choice of $L$ is compatible with the requirement $L \geq 0$ because of the condition $K \geq N / 2$ in Lemma 4.1.

Now any $d_{K}$ in the interval (33) can be expressed as $d_{K}=\lambda d_{K, m}+(1-\lambda) d_{K, M}$ with $0 \leq \lambda \leq 1$. As a result any $D(z)$ with $d_{K}$ in the interval (33) can be expressed as a convex combination

$$
D(z)=\lambda D_{1}(z)+(1-\lambda) D_{2}(z), \quad 0 \leq \lambda \leq 1 .
$$

For an arbitrary $D(z)$ of the form (60), the function $G(z)$ defined in (54) can be written as the convex combination

$$
G(z)=\lambda G_{1}(z)+(1-\lambda) G_{2}(z), \quad 0 \leq \lambda \leq 1
$$

because the denominators of $G(z), G_{1}(z)$ and $G_{2}(z)$ are the same in view of the choice of $L$. Since $G_{1}(z)$ and $G_{2}(z)$ are reactances of degree $N,(61)$ is a reactance (by Corollary following Theorem 5.1). The poles of this reactance are the same as the poles of $G_{1}(z)$ (which in turn are the poles of $G_{2}(z)$ ) and the degree of $G(z)$ is equal to $N$. By using Lemma 5.1 we therefore conclude that $D(z)$ is $\mathrm{SH}$ for all $d_{K}$ in (33) indeed, completing the proof of Lemma 4.1.

Next assume that all the coefficients in (34) are uncertain. It is clear that an arbitrary $D(z)$ with the uncertain coefficients in the range (34) can be obtained from the $L$ vertex polynomials by repeated convex combinations. By repeated application of the argument used in the above proof of Lemma 4.1 , we can then conclude that any $D(z)$ with the coefficients constrained by (34) is SH if (and, trivially, only if) the $L$ vertex polynomials are $\mathrm{SH}$, completing the proof of Lemma 4.2.

\subsection{Proof of Theorem 4.1}

Recall that for the purposes of Theorem 4.1 we started from the coefficients of $D(z)$ and defined two sets of coefficients $p_{n}$ and $q_{n}$ which are coefficients of $P(\omega)$ and $Q(\omega)$ as in (45). These coefficients were assumed to be uncertain as in $(46,47)$. Two vertex polynomials $P_{1}(\omega)$ and $P_{2}(\omega)$ were then defined from which the four polynomials $D_{k n}(z)$ in (52) were obtained. The claim of the Theorem which requires to be proved is that $D(z)$ is SH for every possible values of $p_{n}$ and $q_{n}$ from $(46,47)$ if the four polynomials $D_{k n}(z)$ are $\mathrm{SH}$.

The new polynomials $P(\omega)$ and $Q(\omega)$ can be interpreted in terms of discrete-time reactance language as follows.
Given $D(z)$, suppose we define $G(z)$ as in (54) with $L=0$ :

$$
G(z)=\frac{D(z)+\widehat{D}(z)}{D(z)-\widehat{D}(z)} .
$$

In view of (37) we see that we can also write $G(z)=$ $S(z) / A(z)$, which can be rewritten in terms of $P(\omega)$ and $Q(\omega)$ as

$$
G\left(e^{j \omega}\right)=\frac{P(\omega)}{j \sin (\omega) Q(\omega)}
$$

We-know that $D(z)$ is $\mathrm{SH}$ if and only if $G(z)$ is a reactance of degree $N$. The quantities $P(\omega)$ and $Q(\omega)$ therefore play a role analogous to $P\left(s^{2}\right)$ and $Q\left(s^{2}\right)$ for the continuous-time case in Sec. II. If $G(z)$ is a reactance so that it behaves as in Fig. 7 , then the poles and zeros of $G(z)$ must be single, restricted to be on the unit circle, and must interlace each other.

To prove Theorem 4.1, we first assume that only the coefficients $p_{n}$ are uncertain, and that $q_{n}$ are fixed at some value. We shall prove that if $D_{1}(z)$ and $D_{2}(z)$ defined according to

$$
\begin{aligned}
& D_{1}\left(e^{j \omega}\right)=e^{-j \omega M}\left[P_{1}(\omega)+j \sin (\omega) Q(\omega)\right] \\
& D_{2}\left(e^{j \omega}\right)=e^{-j \omega M}\left[P_{2}(\omega)+j \sin (\omega) Q(\omega)\right]
\end{aligned}
$$

are SH then so is $D(z)$ for any set of $p_{n}$ satisfying (46). Define the functions

$$
G_{1}\left(e^{j \omega}\right)=\frac{P_{1}(\omega)}{j \sin (\omega) Q(\omega)}, \quad G_{2}\left(e^{j \omega}\right)=\frac{P_{2}(\omega)}{j \sin (\omega) Q(\omega)} .
$$

From Lemma 5.1 we know that $G_{1}(z)$ and $G_{2}(z)$ are reactances of degree $N$ if $D_{1}(z)$ and $D_{2}(z)$ are SH. From the definitions $(48,49)$ of $P_{1}(\omega)$ and $P_{2}(\omega)$ and from $(46)$ we see that the following inequality is true:

$$
P_{1}(\omega) \leq P(\omega) \leq P_{2}(\omega)
$$

which is analogous to the sandwich property of (24).

If we let $G_{1}\left(e^{j \omega}\right)=j V_{1}(\omega), G_{2}\left(e^{j \omega}\right)=j V_{2}(\omega)$ and $G\left(e^{j \omega}\right)=j V(\omega)$ then we see that the quantity $V(\omega)$ is sand= wiched between the quantities $V_{1}(\omega)$ and $V_{2}(\omega)$ as demonstrated in Fig. 8, because of property (67) and because of the fact that the denominators of $V(\omega), V_{1}(\omega)$ and $V_{2}(\omega)$ are the same. Summarizing, $V(\omega)$ behaves exactly as it would behave if $G\left(e^{j \omega}\right)$ were a reactance of degree $N$, i.e., if $D(z)$ were SH. This proves that, if $D_{1}(z)$ and $D_{2}(z)$ are $\mathrm{SH}$ then $D(z)$ is $\mathrm{SH}$ for all $p_{n}$ satisfying (46). For convenience of reference we state this as a Lemma.

Lemma 5.2. Suppose $D(z)$ is as in (31) with $N$ even and $d_{N} \neq 0$ so that $D\left(e^{j \omega}\right)$ can be written as in (44) where $P(\omega)$ and $Q(\omega)$ are as in (45). Assume that $q_{n}$ are fixed and $p_{n}$ are uncertain but confined to the interval (46). Define the polynomials $D_{1}(z)$ and $D_{2}(z)$ as in $(64,65)$. Then $D(z)$ is SH for all $p_{n}$ constrained by (46) if and only if $D_{1}(z)$ and $D_{2}(z)$ are $\mathrm{SH}$.

A counterpart of this result when $p_{n}$ are fixed and $q_{n}$ are uncertain is the following. 
Lemma 5.3. Suppose $D(z)$ is as in (31) with $N$ even and $d_{N} \neq 0$ so that $D\left(e^{j \omega}\right)$ can be written as in (44) where $P(\omega)$ and $Q(\omega)$ are as in (45). Assume that $p_{n}$ are fixed and $q_{n}$ are uncertain but confined to the interval (47). Define the polynomials $D_{3}(z)$ and $D_{4}(z)$ as

$$
\begin{aligned}
& D_{3}\left(e^{j \omega}\right)=e^{-j \omega M}\left[P(\omega)+\jmath \sin (\omega) Q_{1}(\omega)\right], \\
& D_{4}\left(e^{j \omega}\right)=e^{-j \omega M}\left[P(\omega)+\jmath \sin (\omega) Q_{2}(\omega)\right],
\end{aligned}
$$

where $Q_{1}(\omega)$ and $Q_{2}(\omega)$ are as in $(50,51)$. Then $D(z)$ is SH for all $q_{n}$ constrained by (47) if and only if $D_{3}(z)$ and $D_{4}(z)$ are $\mathrm{SH}$.

We can combine Lemmas 5.2 and 5.3 (in a manner exactly analogous to the way we combined Lemmas 3.1 and 3.2) to finally arrive at Theorem 41 . Details are omitted to avoid repetitions.

\section{KHARITONOV-TYPE RESULTS FOR STRICTLY-POSITIVE-REAL FUNCTIONS.}

In classical electrical network theory, positive real functions (PR functions) have played a very crucial role $[3],[8]$. A real rational function is 'positive real' if and only if it is the impedance of an $L, C, R$ network with nonnegative element values. Based on the theoretical properties of PR functions, techniques for the synthesis of electrical networks with prescribed impedances have been developed several decades ago [8].

In the modern signal processing era, PR functions still continue to be important, but for a different reason. This is related to the stability of closed loop systems under nonlinear and time-varying conditions. One of the most common system of this type is a time-varying nonlinearity connected to a linear time invariant system $H_{a}(s)$ to form a feed back loop (Fig. 9). The importance of the PR property in connection with the stability of the closed loop system is well-known $[26],[27]$.

There are some algorithms in adaptive signal processing [18],[28] which can be modeled i i the form of a feedback loop with a linear-time invariant feedback path $\left(H_{a}(s)\right.$ or $H(z))$. In these applications it is necessary to ensure that $H_{a}(s)$ (or $H(z)$ as the case may be) is strictly PR, in order for the adaptation algorithm to converge. The coefficients of $H_{a}(s)$ are however uncertain, wh.ich makes this task difficult. In this context, Kharitonov-type results for the PR property (rather than for the SH property) have been found to be useful. We shall now discuss some of these. The results in Sec. 6.1. were reported in 18]. The results in Sec. 6.2 (discrete-time case) are new.

\subsection{Continuous-time SPR Kharitc nov-type theorems.}

A real rational transfer functic $H(s)=\mathcal{N}(s) / D(s)$ is said to be strictly positive real (SP R) if $\mathcal{N}(s)$ and $D(s)$ are $\mathrm{SH}$ and if $\operatorname{Re}[H(j \Omega)]>0$ for all $\Omega$. Votice that $H(s)$ is SPR if and only if $1 / H(s)$ is SPR. Supr ose we assume that the coefficients of $\mathcal{N}(s)$ are fixed and th it of $D(s)$ are uncertain, occupying an interval exactly as in (2). We would like to know whether $H(s)$ is SPR for every choice of $d_{n}$ from (2). Once again this appears to involve the testing of an infinite number of rational functions. It has recently been shown [18] that a Kharitonov type of result exists in this case also. This is stated next.

Theorem 6.1. Suppose $H(s)=\mathcal{N}(s) / D(s)$ is a real rational function with the coefficients of $\mathcal{N}(s)$ fixed. Assume that $D(s)$ is as in (1) where the coefficients $d_{n}$ are uncertain, belonging to the range (2). Define the four Kharitonov polynomials $D_{k n}(s)$ as in (16), and define the four rational functions $H_{k n}(s)=\mathcal{N}(s) / D_{k n}(s)$. Then $H(s)$ is SPR of degree $N$ for all possible choices of $d_{n}$ in the range (2) if and only if all four $H_{k n}(s)$ are SPR of degree $N$.

Results of this type find application in adaptive identification systems [18]. To prove the theorem, first notice that if $H_{k n}(s)$ are SPR of degree $N$ then $D_{k n}(s)$ and $\mathcal{N}(s)$ are SH. This implies, in particular, that $D(s)$ is $\mathrm{SH}$ for all possible $d_{n}$ in (2) (because of Theorem 3.1). The only issue that remains to be proved is that the real part of $H(j \Omega)$ is positive for all $\Omega$ for every possible $D(s)$ with coefficients constrained by (2). Since $\operatorname{Re}[x]>0$ holds if and only if $\operatorname{Re}[1 / x]>0$, we shall work with real part of $G(s) \triangleq 1 / H(s)$ for convenience. We shall again use the decomposition of the form (3) in the proof. This decomposition will be employed both for $\mathcal{N}(s)$ and $D(s)$, so we need a slightly more sophisticated notation. Thus let

$$
D(s)=P_{d}\left(s^{2}\right)+s Q_{d}\left(s^{2}\right)
$$

and

$$
\mathcal{N}(s)=P_{n}\left(s^{2}\right)+s Q_{n}\left(s^{2}\right)
$$

In terms of this representation we have

$G(j \Omega)=\frac{\left[P_{d}\left(\sim \Omega^{2}\right)+j \Omega Q_{d}\left(-\Omega^{2}\right)\right]\left[P_{n}\left(-\Omega^{2}\right)-j \Omega Q_{n}\left(-\Omega^{2}\right)\right]}{|\mathcal{N}(j \Omega)|^{2}}$

so that the real part is positive if and only if the quantity

$$
R(\Omega) \triangleq P_{d}\left(-\Omega^{2}\right) P_{n}\left(-\Omega^{2}\right)+\Omega^{2} Q_{d}\left(-\Omega^{2}\right) Q_{n}\left(-\Omega^{2}\right)
$$

is positive.

Recall that the coefficients of $P_{n}\left(s^{2}\right)$ and $Q_{n}\left(s^{2}\right)$ are fixed and only those of $P_{d}\left(s^{2}\right)$ and $Q_{d}\left(s^{2}\right)$ are uncertain. We shall now play the usual trick employed in Sec. III and again in Sec. 5.4, viz., assume first that the coefficients of $Q_{d}\left(s^{2}\right)$ are fixed and that of $P_{d}\left(s^{2}\right)$ are uncertain and then assume the opposite and finally combine these two. Thus, assuming that $P_{d}\left(s^{2}\right)$ has uncertain coefficients we define the polynomials $P_{d, 1}\left(s^{2}\right)$ and $P_{d, 2}\left(s^{2}\right)$ exactly as in $(12,13)$. For any $P_{d}\left(s^{2}\right)$ with coefficients bounded in a manner analogous to (17) we once again have the familiar sandwich property

$$
P_{d, 1}\left(-\Omega^{2}\right) \leq P_{d}\left(-\Omega^{2}\right) \leq P_{d, 2}\left(-\Omega^{2}\right) .
$$


If we assume that $H(s)$ is SPR with the two extreme choices of $P_{d}\left(s^{2}\right)$, viz., $P_{d}\left(s^{2}\right)=P_{d, 1}\left(s^{2}\right)$ and with $P_{d}\left(s^{2}\right)=$ $P_{d, 2}\left(s^{2}\right)$ we have

$$
\begin{aligned}
& P_{d, 1}\left(-\Omega^{2}\right) P_{n}\left(-\Omega^{2}\right)+\Omega^{2} Q_{d}\left(-\Omega^{2}\right) Q_{n}\left(-\Omega^{2}\right)>0 \\
& P_{d, 2}\left(-\Omega^{2}\right) P_{n}\left(-\Omega^{2}\right)+\Omega^{2} Q_{d}\left(-\Omega^{2}\right) Q_{n}\left(-\Omega^{2}\right)>0
\end{aligned}
$$

Because of (74) we can express $P_{d}\left(-\Omega^{2}\right)$ as a convex combination

$$
P_{d}\left(-\Omega^{2}\right)=\lambda(\Omega) P_{d, 1}\left(-\Omega^{2}\right)+(1-\lambda(\Omega)) P_{d, 2}\left(-\Omega^{2}\right)
$$

where $0 \leq \lambda(\Omega) \leq 1$. If we multiply both sides of (75) by $\lambda(\Omega)$ the inequality is preserved (because $\lambda(\Omega) \geq 0$ ). Same is true if we multiply both sides of (76) with $1-\lambda(\Omega)$. Adding these two inequalities after such multiplication and using (77) we obtain

$$
P_{d}\left(-\Omega^{2}\right) P_{n}\left(-\Omega^{2}\right)+\Omega^{2} Q_{d}\left(-\Omega^{2}\right) Q_{n}\left(-\Omega^{2}\right)>0
$$

which proves that the real part of $G(j \Omega)$ is positive for all $\Omega$ for any choice of the coefficients of $P_{d}\left(s^{2}\right)$ from the interval of uncertainty. A similar result follows if we assume that the coefficients of $P_{d}\left(s^{2}\right)$ are fixed and that of $Q_{d}\left(s^{2}\right)$ are uncertain. By combining these in the usual manner, one can then conclude that the real part of $G(j \Omega)$ (and hence that of $H(j \Omega)$ ) is positive for all values of the coefficients of $D_{d}(s)$ in the uncertain interval. This concludes the proof of Theorem 6.1.

If the coefficients of $\mathcal{N}(s)$ and $D(s)$ are both uncertain, a similar result can easily be obtained, involving sixteen (rather than four) tests. See [25] for details.

6.2 Discrete-time SPR Kharitonov-type theorems.

Let $H(z)=\mathcal{N}(z) / D(z)$ be a transfer function with

$$
\mathcal{N}(z)=\sum_{i=0}^{N} n_{i} z^{-i}, \quad D(z)=\sum_{i=0}^{N} d_{i} z^{-i},
$$

where the coefficients are real. The definition of SPR property is analogous to that in Sec. 6.1, i.e., $H(z)$ is said to be SPR if $\mathcal{N} z$ ) and $D(z)$ are SH and $\operatorname{Re}\left[H\left(e^{j \omega}\right)\right]>0$ for all real $\omega$. Notice that $H(z)$ is SPR if and only if $1 / H(z)$ is SPR. W' hen the coefficients $d_{i}$ and/or $n_{i}$ are uncertain, we have an infinitely large family of rational functions. We would like to know whether all these functions are SPR, based on a finite number of tests. We shall now present some Kharitonov-type theorems for this purpose.

Theorem 6.2. Let $H(z)=\mathcal{N}(z) / D(z)$ be real rational with $\mathcal{N}(z)$ and $D(z)$ as in (79). Suppose that the numerator coefficients are fixed, and that only the denominator coefficients $d_{i}$ for $i \geq N / 2$ are uncertain as in (34) so that there are $L$ uncertain coefficients, with $L$ defined by (35). Define the $2^{L}$ vertex polynomials $D_{k}(z)$ as in Lemma 4.2. Let $\mathcal{N}(z)$ and the $2^{L}$ vertex polynomials $D_{k}(z)$ be SH. Then
$H(z)$ is SPR for every possible value of the uncertain coeffcients satisfying (34) if and only if the $2^{L}$ rational functions $H_{k}(z)=\mathcal{N}(z) / D_{k}(z)$ are SPR.

If the coefficients of $\mathcal{N}(z)$ are also uncertain, we can develop a similar theorem, but we have to test a larger number of polynomials. More specifically, we have the following result.

Theorem 6.3. Assume that the conditions of Theorem 6.2 prevail with the exception that the $L$ coefficients of $n_{i}$ are also uncertain belonging to the regions

$$
n_{i, m} \leq n_{i} \leq n_{i, M}, \quad N / 2 \leq i \leq N .
$$

where $L$ is as in (35). Suppose that the $2^{L}$ vertex polynomials $D_{k}(z)$ and $2^{L}$ vertex polynomials $N_{\ell}(z)$ with $1 \leq k, \ell \leq$ $2^{L}$ are SH. Then $H(z)$ is SPR for all possible combinations of the uncertain coefficients $d_{i}$ in (34) and $n_{i}$ in (80) if and only if the $2^{2 L}$ rational functions $H_{k \ell}(z)=\mathcal{N}_{k}(z) / D_{\ell}(z)$, $1 \leq k, \ell \leq 2^{L}$, are SPR.

According to these theorems, the number of rational functions to be tested grows exponentially with $N$. We now obtain a result in which the number of tests does not grow with $N$. In order to arrive at such a test, we shall express $D\left(e^{j \omega}\right)$ and $\mathcal{N}\left(e^{j \omega}\right)$ in terms of their $P(\omega)$ and $Q(\omega)$ components as in (45). Assume that $N$ is even (for odd $N$, a similar developement is possible) so that $D\left(e^{j \omega}\right)$ and $\mathcal{N}\left(e^{j \omega}\right)$ can be expressed as

$$
\begin{aligned}
& D\left(e^{j \omega}\right)=e^{-j \omega M}[P(\omega)+j \sin (\omega) Q(\omega)], \\
& \mathcal{N}\left(e^{j \omega}\right)=e^{-j \omega M}\left[P^{\prime}(\omega)+j \sin (\omega) Q^{\prime}(\omega)\right],
\end{aligned}
$$

where

$$
\begin{aligned}
P(\omega) & =\sum_{n=0}^{M} p_{n} \cos ^{2 n}(\omega / 2), & Q(\omega) & =\sum_{n=0}^{M-1} q_{n} \cos ^{2 n}(\omega / 2), \\
P^{\prime}(\omega) & =\sum_{n=0}^{M} p_{n}^{\prime} \cos ^{2 n}(\omega / 2), & Q^{\prime}(\omega) & =\sum_{n=0}^{M-1} q_{n}^{\prime} \cos ^{2 n}(\omega / 2) .
\end{aligned}
$$

Assume that $p_{n}, q_{n}, p_{n}^{\prime}$ and $q_{n}^{\prime}$ are uncertain, and belong to the intervals

$$
\begin{gathered}
p_{n, m} \leq p_{n} \leq p_{n, M}, \quad 0 \leq n \leq M, \\
q_{n, m} \leq q_{n} \leq q_{n, M}, \quad 0 \leq n \leq M-1, \\
p_{n, m}^{\prime} \leq p_{n}^{\prime} \leq p_{n, M}^{\prime}, \quad 0 \leq n \leq M, \\
q_{n, m}^{\prime} \leq q_{n}^{\prime} \leq q_{n, M}^{\prime}, \quad 0 \leq n \leq M-1 .
\end{gathered}
$$

Define the four polynomials $D_{k n}, 1 \leq k, n \leq 2$ and the four polynomials $N_{i \ell}(z), 1 \leq i, \ell \leq 2$, as

$$
D_{k n}\left(e^{j \omega}\right)=e^{-j \omega M}\left[P_{k}(\omega)+j \sin (\omega) Q_{n}(\omega)\right]
$$

and

$$
\mathcal{N}_{i \ell}\left(e^{j \omega}\right)=e^{-j \omega M}\left[P_{i}^{\prime}(\omega)+j \sin (\omega) Q_{\ell}^{\prime}(\omega)\right]
$$


We can therefore define the sixteen real rational functions

$$
\frac{N_{i \ell}(z)}{D_{k n}(z)}, \quad 1 \leq i, \ell, k, n \leq 2
$$

We now claim the following result.

Theorem 6.4. With the above setup, let the four polynomials $D_{k n}(z)$ and the four polynomials $\mathcal{N}_{i \ell}(z)$ be SH and let the sixteen rational functions defined in $(86)$ be $S P R$. Then every rational function $\mathcal{N}(z) / D(z)$ which satisfies (85) is SPR.

6.3 Proofs of Discrete-time SPR Kharitonov-type theorems.

First consider Theorem 6.2 where only the $d_{n}$ coefficients with $n \geq N / 2$ are uncertain. We begin by assuming that only one coefficient, say $d_{K}$ with $K \geq N / 2$, is uncertain so that

$$
d_{K, m} \leq d_{K} \leq d_{K, M}
$$

We know from Lemma 4.1 that if the two vertex polynomials $D_{1}(z)$ and $D_{2}(z)$ (which correspond to $d_{K}=d_{K, m}$ and $d_{K}=d_{K, M}$ respectively) are SH then $D(z)$ is $\mathrm{SH}$ for every $d_{K}$ satisfying (87). According to the conditions of the Theorem $6.2, \mathcal{N}(z), D_{1}(z)$ and $D_{2}(z)$ are $\mathrm{SH}$, so that in order to prove the theorem it only remains to prove that $\left.\operatorname{Re}\left[H e^{j \omega}\right)\right]>0$ if $\operatorname{Re}\left[H_{1}\left(e^{j \omega}\right)\right]>0$ and $\left.\operatorname{Re}\left[H_{2} e^{j \omega}\right)\right]>0$. Define $G(z)=1 / H(z), G_{1}(z)=1 / H_{1}(z)$ and $G_{2}(z)=1 / H_{2}(z)$ for convenience. Since $\operatorname{Re}[x]>0$ if and only if $\operatorname{Re}[1 / x]>0$, it remains only to prove that $\operatorname{Re}\left[G\left(e^{j \omega}\right)\right]>0$ for any value of the uncertain coefficient $d_{K}$ in (87) if $\operatorname{Re}\left[G_{1}\left(e^{j \omega}\right)\right]$ and $\operatorname{Re}\left[G_{2}\left(e^{j \omega}\right)\right]$ are positive.

The strategy now is to define the two polynomials

$$
\begin{aligned}
& S(z)=D(z)+z^{-L} \hat{D}(z) \\
& A(z)=D(z)-z^{-L} \hat{D}(z) .
\end{aligned}
$$

Clearly the coefficients of $S(z)$ and $A(z)$ are symmetric and antisymmetric respectively, with center of symmetry $M \triangleq(N+L) / 2$. Moreover $D(z)$ can be expressed as

$$
D(z)=\frac{1}{2}[S(z)+A(z)] .
$$

if we choose $L=2 K-N$ then the center $M$ coincides with $\bar{K}$ so that the antisymmetric polynomial $A(z)$ works out to be the same for every possible value of $d_{K}$ in (87). Since every $d_{K}$ in (87) can be expressed as a convex combination of $d_{K, m}$ and $d_{K, M}$, we can express $S(z)$ in (88) as

$$
S(z)=\lambda S_{1}(z)+(1-\lambda) S_{2}(z), \quad 0 \leq \lambda \leq 1,
$$

for every $D(z)$ with $d_{K}$ satisfying (87).

Having expressed $D(z)$ as in (89), we can express $\mathcal{N}(z)$ as

$$
N(z)=\frac{1}{2}\left[S^{\prime}(z)+A^{\prime}(z)\right]
$$

where

$$
S^{\prime}(z)=\mathcal{N}(z)+z^{-L^{\prime}} \hat{\mathcal{N}}(z)
$$

$$
A^{\prime}(z)=\mathcal{N}(z)-z^{-L^{\prime}} \hat{\mathcal{N}}(z)
$$

where $L^{\prime}$ is chosen so that the center of symmetry of $S^{\prime}(z)$ coincides with the center $M$ of $S(z)$. (Since the degrees of $D(z)$ and $\mathcal{N}(z)$ are assumed to be the same, $L^{\prime}=L$ works. Otherwise a different $L^{\prime}$ has to be used.) With these definitions we now have

$$
\begin{array}{rlrl}
S\left(e^{j \omega}\right) & =e^{-j \omega M} P(\omega), & A\left(e^{j \omega}\right) & =j e^{-j \omega M} Q(\omega), \\
S^{\prime}\left(e^{j \omega}\right) & =e^{-j \omega M} P^{\prime}(\omega), \quad A^{\prime}\left(e^{j \omega}\right)=j e^{-j \omega M} Q^{\prime}(\omega),
\end{array}
$$

where $P(\omega), Q(\omega), P^{\prime}(\omega)$ and $Q^{\prime}(\omega)$ are real functions of $\omega$. The real part of $G\left(e^{j \omega}\right)$ can now be expressed as

$$
\operatorname{Re}\left[G\left(e^{j \omega}\right)\right]=\frac{P(\omega) P^{\prime}(\omega)+Q(\omega) Q^{\prime}(\omega)}{|\mathcal{N}|^{2}} .
$$

It therefore only remains to prove that

$$
P(\omega) P^{\prime}(\omega)+Q(\omega) Q^{\prime}(\omega)>0
$$

under the conditions that

$$
\begin{aligned}
& P_{1}(\omega) P^{\prime}(\omega)+Q(\omega) Q^{\prime}(\omega)>0 \\
& P_{2}(\omega) P^{\prime}(\omega)+Q(\omega) Q^{\prime}(\omega)>0 .
\end{aligned}
$$

This is easily done by noticing that in view of $(90) P(\omega)$ can be expressed as a convex combination

$$
P(\omega)=\lambda P_{1}(\omega)+(1-\lambda) P_{2}(\omega), \quad 0 \leq \lambda \leq 1 .
$$

Since (96) obviously holds for $\lambda=0$ (because of (98)) and for $\lambda=1$ (because of (97)), let us prove (96) only for the case $0<\lambda<1$. Multiplying the LHS of (97) by $\lambda$ does not affect the inequality (because $\lambda>0$ ) and similarly multiplying the LHS of (98) by $(1-\lambda)$ does not affect the inequality. Adding these, we therefore arrive at (96) which proves that $\operatorname{Re}\left[G\left(e^{j \omega}\right)\right]$ and hence $\operatorname{Re}\left[H\left(e^{j \omega}\right)\right]$ are positive for all $\omega$, as long as $\operatorname{Re}\left[H_{1}\left(e^{j \omega}\right)\right]$ and $\operatorname{Re}\left[H_{2}\left(e^{j \omega}\right)\right]$ are positive for all $\omega$. This in essence completes the proof of Theorem 6.2 under the assumption that only one coefficient $d_{K}$ is uncertain with $K \geq N / 2$. If all the coefficients $d_{i}, N / 2 \leq i \leq N$ are uncertain, then by judicious repetition of the above argument, one can easily verify the truth of the Theorem.

Next consider Theorem 6.3. This can be proved with the help of Theorem 6.2 as follows. We have $2^{L}$ vertex polynomials $\mathcal{N}_{\ell}$ for the numerator and $2^{L}$ vertex polynomials $D_{k}(z)$ for the denominator. For a given $\ell$, consider the set $\mathcal{N}_{\ell}(z) / D_{k}(z)$ for $1 \leq k \leq 2^{L}$. Since these are SPR, we can use Theorem 6.2 to conclude that $\mathcal{N}_{\ell}(z) / D(z)$ is SPR as long as the uncertain coefficients of $D(z)$ satisfy (34). This means that the function $G(z)=D(z) / \mathcal{N}_{\ell}(z)$ is SPR for any $D(z)$ whose uncertain coefficients satisfy (34), for any vertex polynomial $N_{\ell}(z)$. By applying Theorem 6.2 again we see that the ratio $D(z) / \mathcal{N}(z)$ (and hence $\mathcal{N}(z) / D(z)$ ) is SPR for any $D(z)$ and for any $\mathcal{N}(z)$ with the uncertain coefficients constrained as in (34),(80). This proves Theorem 6.3 . 
Finally we come to Theorem 6.4. We can define the vertex functions $P_{1}(\omega), P_{2}(\omega), Q_{1}(\omega)$ and $Q_{2}(\omega)$ as in (48-51), and similarly define vertex functions for $P^{\prime}(\omega)$ and $Q^{\prime}(\omega)$. Sandwich properties of the form (67) are then satisfied by the functions $P(\omega), Q(\omega), P^{\prime}(\omega)$ and $Q^{\prime}(\omega)$.

In order to prove the theorem we note that

$$
\operatorname{Re}\left[G\left(e^{j \omega}\right)\right]=\frac{P(\omega) P^{\prime}(\omega)+\sin ^{2}(\omega) Q(\omega) Q^{\prime}(\omega)}{\left|\mathcal{N}\left(e^{j \omega}\right)\right|^{2}} .
$$

Assuming that this quantity is positive for all the sixteen possible combinations of the vertex functions $P_{k}(\omega), Q_{\ell}(\omega)$, $P_{i}^{\prime}(\omega)$ and $Q_{j}^{\prime}(\omega), 1 \leq k, \ell, i, j \leq 2$, it is a simple matter to prove that $(100)$ is positive for any possible set of values of the coefficients in (85). This proves that the real part of $H\left(e^{j \omega}\right)$ is positive for all possible values of the coefficients in (85). By using Theorem 4.1 we can also prove that $\mathcal{N}(z)$ and $D(z)$ are SH under the conditions of Theorem 6.4 as long as the coefficients are constrained by (85). This then concludes the proof that $H(z)$ is SPR for all values of the coefficients from the range (85). Details are omitted because these can be easily worked out.

In Theorems 6.2-6.4, the degrees of $\mathcal{N}(z)$ and $D(z)$ are assumed to be equal for simplicity. If this is not the case, the Theorems continue to hold after some simple adjustments. It should be noted that unlike in the continuoustime case, a discrete-time SPR function can have unrelated degrees for $\mathcal{N}(z)$ and $D(z)$. Here is an extreme example of a SPR function with denominator of degree zero and numerator of arbitrary degree $K: H(z)=2+z^{-K}$.

\section{OTHER GENERALIZATIONS}

There are several other extensions of Kharitonov's theorem which we have not discussed in this paper. One of these is for the case of continuous time systems with complex coefficients. In this case a result similar to Kharitonov's theorem of Sec. III can be established for the SH property, expect that we need more thān 4 tests. Specific details can be found in [5], which includes proofs of a 16-point test and a 8-point test. Secondly, Kharitonov's results have been extended to the case of multivariable polynomials, i.e., polynomials which are functions of more than one variable $s$. Details of this can be found in [25] and references therein.

\section{References.}

[1] A. V. Oppenheim, A. S. Willsky and I. T. Young, Signals and systems, Prentice Hall, Inc., 1983.

[2] B. C. Kuo, Automatic control systems, Prentice Hall, 1976.

[3] N. Balabanian and T. Bickart, Electrical network theory, John Wiley and Sons, Inc., 1969.

[4] V. L. Kharitonov, "Asymptotic stability of an equilibrium position of a family of systems of linear differential equations," Differential Equations, vol. 14, pp. 14831485,1979
5] N. K. Bose and Y. Q. Shi, "A simple general pro of of Kharitonov's generalized stability criterion," IEEE Trans. CAS, pp. 1233-1237, Oct. 1987.

6] C. V. Hollot and A. C. Bartlett, "Some discrete-time counterparts to Kharitonov's stability criterion for uncertain systems," IEEE Trans. AC, pp. 355-356, Apr. 1986.

[7] J. Cieslik, "On the possibilities of the extension of Kharitonov's stability test for interval polynomials to the discrete-time case," IEEE Trans. AC, pp. 237-238, Mar. 1987.

[8] E. A. Guillemin, Synthesis of passive networks, John Wiley \& sons, Inc., 1957.

[9] A. V. Oppenheim and R. W. Schafer, Digital signal processing, Prentice Hall, Inc. 1975.

[10] E. I. Jury, Theory and application of the $z$-transform method, Wiley, NY, 1964.

[11] A. Antoniou, Digital filters: analysis and synthesis, McGraw Hill Book Co., 1979.

[12] P. P. Vaidyanathan and S. K. Mitra, "A unified structural interpretation of some well-known stability-test procedures for linear systems," Proc. of the IEEE, pp. 478-497, April 1987.

[13] L. R. Rabiner and B. Gold, Theory and Application o: digital signal processing, Prentice Hall, Inc., 1975.

114 P. P. Vaidyanathan, "Design and implementation of digital FIR filters," in Handbook of digital signal processing, ed. D. F. Elliot, Academic Press, Inc., 1987.

[15] M. E. Van Valkenburg, Modern network synthesis, Johr Wiley \& Sons, Inc., 1964.

[16] H. W. Schuessler, "A stability theorem for discrete systems," IEEE Trans. ASSP, pp. 87-89, Feb. 1976.

[17] P. P. Vaidyanathan and S. K. Mitra, "Synthesis of arbitrary digital transfer functions using allpass-based structures derived via $L B R$ two-pair extraction procedure," Circuits and Signal Processing, vol. 5, No. 3, pp. 343-370, 1986.

[18] S. Dasgupta and A. S. Bhagwat, "Conditions for designing strictly positive real transfer functions for adaptive output error identification," IEEE Trans. on CAS, pp. 731-736, July 1987.

[19] B. R. Barmish, "Invariance of the strict Hurwitz property for polynomials with perturbed coefficients," IEEE Trans. AC, pp. 935-936, Oct. 1984.

(20) N. K. Bose and Y. Q. Shi, "Network realizability theory approach to stability of complex polynomials," IEEE Trans. on CAS, pp. 216-218, Feb. 1987.

[21] K. S. Yeung and S. S. Wang, "A simple proof of Kharitonov's theorem, " IEEE Trans. AC, pp. 822-823, Sept. 1987.

[22] K. H. Wei and R. K. Yedavalli, "Invariance of strict Hurwitz property for uncertain polynomials with dependent coefficients," IEEE Trans. AC, pp. 907-909. Oct. 1987. 
[23] B. D. O. Anderson, E. I. Jury and M. Mansour, "On robust Hurwitz polynomials," IEEE Trans. AC, pp. 909-913, Oct. 1987.

[24] M. B. Argoun, "Frequency domain conditions for the stability of perturbed polynomials," IEEE Trans. AC, pp. 913-916, Oct. 1987.

[25] N. K. Bose, "Multidimensional systems: present state and future prospects," Proc. IEEE Int. Symp. Circ. and Sys., pp. 89-92, Espoo, Finland, June 1988.

[26] L. Hitz and B. D. O. Anderson, "Discrete positive real functions and their application to system stability," Proc. IEE vol. 116, no. 1, pp. 153-155, Jan 1969.

[27] M. Vidyasagar, "Nonlinear systems analysis," Prentice Hall, Inc., 1978.

[28] M. G. Larimore, J. R. Treichler, and C. R. Johnson, "SHARF: an algorithm for adapting IIR digital filters," IEEE Trans. ASSP, pp. 428-440, August 1980.

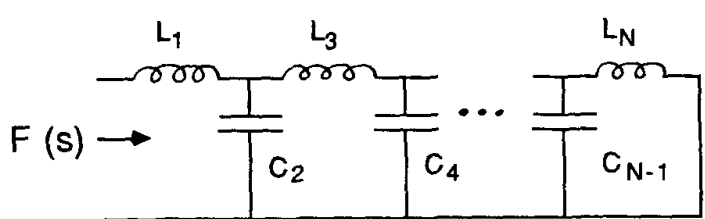

Fig. 1. The LC ladder network associated with a continuedfraction expansion.

\begin{tabular}{lll}
\hline 2 & 8 & 6 \\
1 & 2 & \\
4 & 6 & \\
$\frac{1}{2}$ & & \\
6 & & \\
\hline
\end{tabular}

Fig. 2. Routh-Hurwitz array for the example.

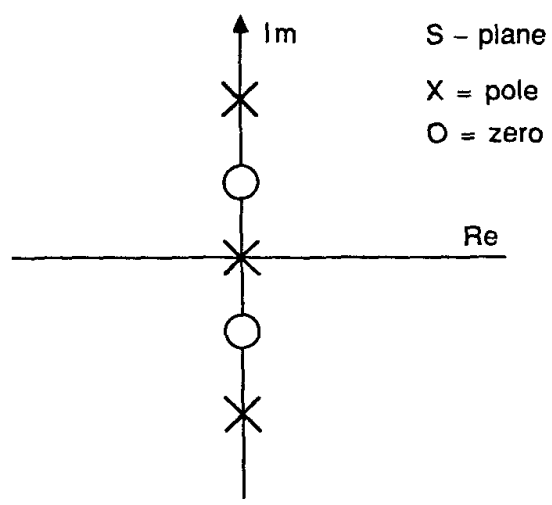

Fig. 3. Demonstration of the pole-zero interlacing property of a reactance $F(s)$.

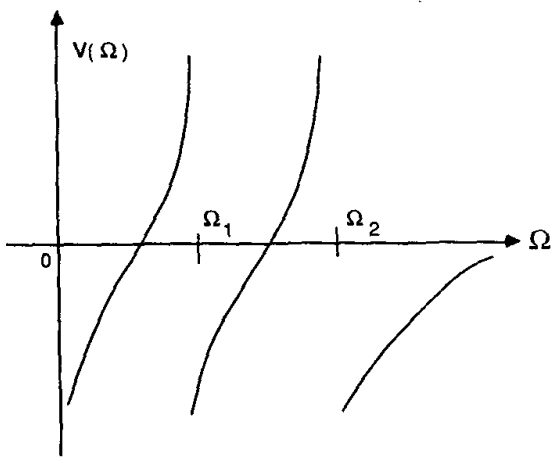

Fig. 4. A typical plot of $V(\Omega)$, where $F(j \Omega)=j V(\Omega)$ is a reactance of degree five. Since $V(\Omega)=-V(-\Omega)$, plot for $\Omega<0$ is not shown.

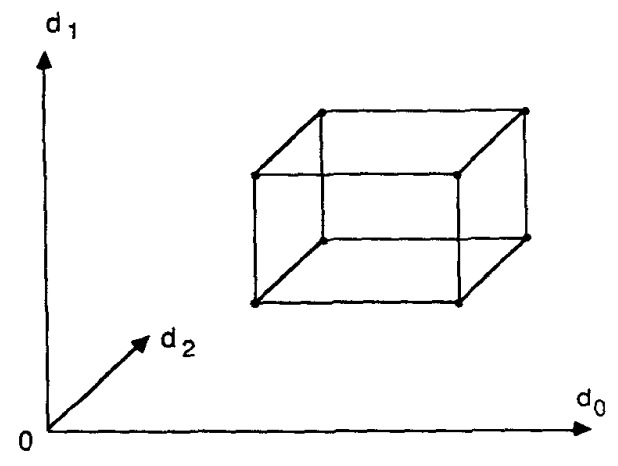

Fig. 5. Demonstration of the eight vertex-polynomials for $N=2$. Here $D(s)=d_{0}+d_{1} s+d_{2} s^{2}$.

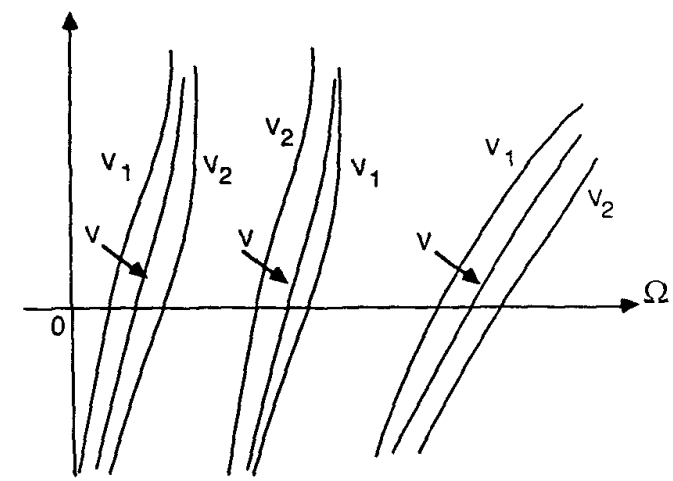

Fig. 6. Typical plots of $V_{1}(\Omega), V_{2}(\Omega), V(\Omega)$ demonstrating the sandwich property. 

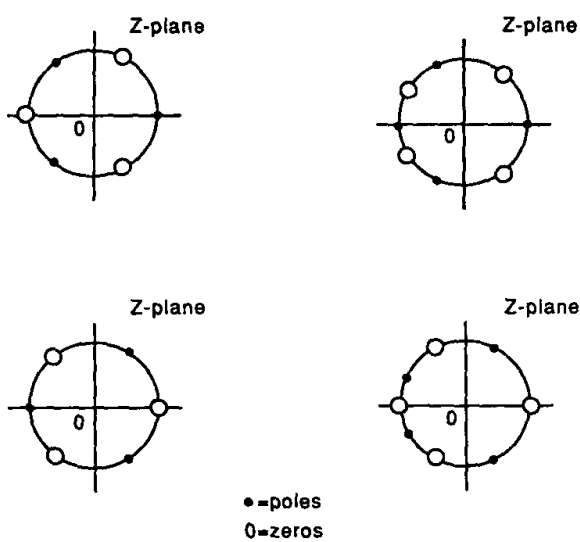

Fig. 7 (a) Demonstrations of the pole-zero alternation property of a discrete time reactance. The circle shown is the unitcircle in the z-plane.

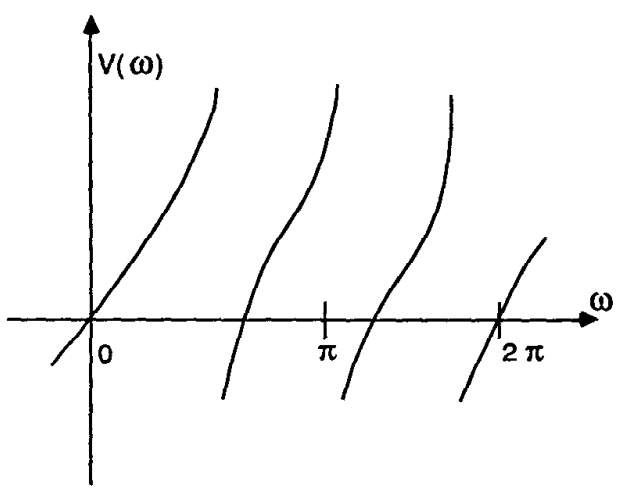

Fig. 7 (b) Demonstration of the alternation property of a reactance $G(z)$. Here $G\left(\theta^{j \omega}\right)=j V(\omega)$.

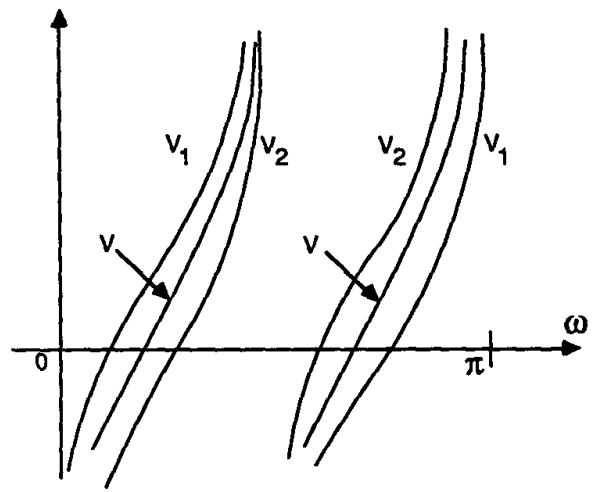

Fig. 8. Demonstrating the sandwich property of $V_{1}(\omega), V_{2}(\omega)$ and $V(\omega)$ in the discrete-time case.

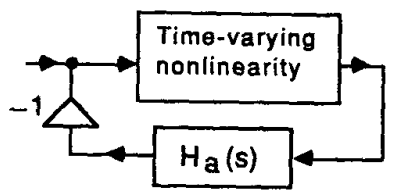

Fig. 9. A closed-loop system with a linear timeinvariant feedback path. 